\title{
Calculation of Aerodynamic Performance Characteristics of Airplane Wing and Comparing with the Experimental Measurement
}

\author{
Haci Sogukpinar* $\ddagger$ and Ismail Bozkurt** \\ *Department of Energy Systems Engineering, Faculty of Technology, University of Adiyaman, Adiyaman 02040, Turkey, \\ **Department of Mechanical Engineering, Faculty of Engineering, University of Adiyaman, Adiyaman 02040, Turkey. \\ (hsogukpinar@adiyaman.edu.tr, ibozkurt@adiyaman.edu.tr)
}

\$Corresponding Author; Hacı Soğukpınar, Department of Energy Systems Engineering, Faculty of Technology, University of Adiyaman, Adiyaman 02040, Turkey, Tel: +90 41622338 00/2840, hsogukpinar@adiyaman.edu.tr

Received: 11.06.2015 Accepted:21.06.2015

\begin{abstract}
With the flight of Wright brothers with the first airplane, it has been begun to design different wing section. With the beginning of the new industrial process, hundreds of aerofoils were designed and implemented in the aviation sector. In this study, aerodynamic performance of airplane NACA 2415 aerofoils was numerically investigated to understand effect of angle of attack on the aerodynamic performance. The lift and drag coefficient, lift to drag ratio and power coefficients around NACA 2415 aerofoil were calculated with SST turbulence model and are compared with two different experimental data to validate simulation accuracy of Computational Fluid Dynamics approach. Result indicates that at the low angle of attack, the lift coefficient of NACA 2415 aerofoil obtained in the present study is in a good agreement with experimental results but after zero degree, agreement is not good with experimental results.
\end{abstract}

Keywords-Airplane, airfoil, aerodynamic performance

\section{Introduction}

It is right to claim that airfoil section of plane is the second most important parameters after wing planform area. The lower and upper surfaces are responsible for creating the ideal pressure distribution. The main task of the wing is to create a lifting force. There is a need for specially designed wing shape to the formation of this force and these are called airfoil. Airfoil design is complex and requires significant expertise because it is a very time consuming process. That's why companies like Boeing and Airbus are designed individually for each aircraft airfoil with their own expert staff. But smaller companies are generally use currently available airfoil shape. Airfoils in many different structures were designed by NACA. National advisory Committee for Aeronautics (NACA)- predecessor of present National Administration for Aeronautics and Astronautics (NASA). They are currently available in several books and websites. These airfoils are used as helicopter blades, propeller blades, hydrofoils, and wind turbine blades are just some examples. Efficiencies of all of these blades rely heavily on the airfoil section. With the advent of high speed and powerful computer, there has been developed airfoil design code that is based on conformal mapping. One of the oldest and most reliable of these codes is Eppler code. That is the reason why two reliable airfoil resources are NACA and Eppler.

Sogukpinar et. al [1] simulated air flow around inclined NACA 632-215 airfoil using SST turbulence model. Lift, drag coefficient, lift to drag ratio and power coefficient around the airfoil were calculated and compared with different velocity. With the increasing of wind velocity, lift and drag coefficient increases, after reaching maximum lift to drag ratio at 4 degree lift to drag ratio starts to degrease again. Mehrdad Ghods [2] experimentally observed lift and drag coefficients of the NACA 2415 airfoil in a wind tunnel. According to the test results, lift coefficient increases as the angle of attack increases between -5 and +17 degrees and at +17 degrees maximum lift was generated. If the angle of attack is increased any further, drag becomes the dominant factor and the wing enters the stall mode. As comparing the theoretical data with experiment, it was observed experimentally a higher drag coefficient. This is because imperfection around airfoil section and experimental inaccuracies. Rajakumar et. al [3] investigated aerodynamic performance characteristics of horizontal-axis wind turbines. The airfoils NACA 4410 and NACA 2415 are taken into consideration for evaluating this proposed approach. It was shown that the approach used in calculation is efficient and 
time saving as compared with the commonly used iterative procedures. The results of computational fluid dynamics (CFD) simulations in two and three spatial dimensions were compared to pressure measurements and particle image velocimetry (PIV) flow surveys to assess the suitability of numerical models for the simulation of deep dynamic stall experiments carried out on a pitching NACA 23012 airfoil [4]. The comparison of the airloads curves and of the pressure distribution over the airfoil surface shows that a three-dimensional numerical model can better reproduce the flow structures and the airfoil performance for the deep dynamic stall regime. A NACA 4415 airfoil model was tested in the Ohio State University Aeronautical and Astronautical Research Laboratory $3 \times 5$ subsonic wind tunnel under steady state and unsteady conditions [5]. Steady state results of the NACA 4415 testing at Reynolds number of 1 million showed a baseline maximum lift coefficient of 1.35 at $14.3^{\circ}$ angle of attack. The numerical simulation of horizontal axis wind turbines (HAWTs) with untwisted blade was performed to determine the optimal angle of attack that produces the highest power output [6]. The power outputs reach maximum at pitch angles: $4.12^{\circ}, 5.28^{\circ}, 6.66^{\circ}$ and $8.76^{\circ}$ for the wind speeds 7.2, 8.0, 9.0 and $10.5 \mathrm{~m} / \mathrm{s}$, respectively.

In this study, NACA 2415 airfoil was simulated with low speed airflow to determine optimal angle of attack for airplane wing. Numerical results of original airfoil were compared with experimental data to validate the calculation accuracy of the CFD. Numerical experiment were conducted by varying the pitch angles: $-4^{\circ},-2^{\circ}, 0^{\circ}, 2^{\circ}, 4^{\circ}, 6^{\circ}, 8^{\circ}, 10^{\circ}$ for the wind speed $19.6 \mathrm{~m} / \mathrm{s}$. this study indicates that maximum lift to drag ratio reaches at the angle of $4^{\circ}$.

\section{Materials and methods}

The equations solved by the Turbulent Flow, SST interface are the Navier-Stokes equation for conservation of momentum and the continuity equation for conservation of mass. The SST model is a so-called low-Reynolds number model, which means that it resolves the flow all the way down to the wall. SST turbulence model is expressed in terms of $\mathrm{k}$ and $\omega$ with equation (1) and (2) [7].

$\rho \frac{\partial k}{\partial t}+\rho u \cdot \nabla k=P-\rho \beta_{0}{ }^{*} k \omega+\nabla \cdot\left(\left(\mu+\sigma_{k} \mu_{T}\right) \nabla_{k}\right)$

$\rho \frac{\partial \omega}{\partial t}+\rho u . \nabla \omega=\frac{\rho \gamma}{\mu_{T}} P-\rho \beta \omega^{2}+\nabla \cdot\left(\left(\mu+\sigma_{\omega} \mu_{T}\right) \nabla_{\omega}\right)+$

$2\left(1-f_{v 1}\right) \frac{\rho \sigma_{\omega^{2}}}{\omega} \nabla \omega . \nabla k$

Where, $\rho$ is density, $\mathrm{u}$ is velocity field of wind, $\mathrm{k}$ is turbulent kinetic energy, $\mu$ is dynamic viscosity of air, $\omega$ is specific dissipation rate, $\beta_{0}{ }^{*}, \sigma_{k}, \beta, \sigma_{\omega}$ are turbulence model parameters. $\mathrm{P}$ is the static pressure and expressed with the equation (3):

$P=\min \left(P_{k}, 10 \rho \beta_{0}{ }^{*} k \omega\right)$

Where, $\mathrm{P}_{\mathrm{k}}$ is production term. The interpolation functions $\mathrm{f}_{\mathrm{v} 1}$ and $f_{\mathrm{v} 2}$ are represented with the equation (4) and (5):

$$
\begin{aligned}
& f_{v 1}= \\
& \tanh \left(\min \left[\max \left(\frac{\sqrt{k}}{\beta_{0}{ }^{*} \omega l_{\omega}}, \frac{500 \mu}{\rho \omega l_{\omega}{ }^{2}}\right), \frac{4 \rho \sigma_{\omega^{2}} k}{\max \left(\frac{2 \rho \omega^{2}}{\omega} \nabla \omega \cdot \nabla k, 10^{-10}\right) l_{\omega}{ }^{2}}\right]^{4}\right) \\
& f_{v 2}=\tanh \left(\max \left(\frac{\sqrt{k}}{\beta_{0}{ }^{*} \omega l_{\omega}}, \frac{500 \mu}{\rho \omega l_{\omega}{ }^{2}}\right)^{2}\right)
\end{aligned}
$$

Where I is intensity and COMSOL default model constants are given by,

$$
\begin{gathered}
\beta_{1}=0.075, \gamma_{1}=\frac{5}{9}, \sigma_{k 1}=0.85, \sigma_{\omega 1}=0.5, \beta_{2}=0.0828 \\
\gamma_{2}=0.44, \sigma_{k 2}=1.0, \sigma_{w 2}=0.856, \beta_{0}{ }^{*}=0.09, \sigma_{1}=0.31 .
\end{gathered}
$$

As shown in Figure 1, computational domain consist of a semicircle and rectangular domain which is created with COMSOL. The inlet port is set as a velocity inlet and values are given in Table 1. Outlet port is set as open boundary and no-slip condition is applied for the blade surface. The computational conditions are shown in Table 1.

Table 1. Computational condition

\begin{tabular}{ll}
\hline Density & $1.20 \mathrm{~kg} / \mathrm{m}^{3}$ \\
Wind speed & $25 \mathrm{~m} / \mathrm{s}, 19.6 \mathrm{~m} / \mathrm{s}$ \\
Angle of attack (Deg) & $-4,-2,0,2,4,6,8,10$ \\
Turbulent kinetic energy & $2.090 \mathrm{E}-7 \mathrm{~m}^{2} / \mathrm{s}^{2}$ \\
Specific dissipation rate & $1.38891 / \mathrm{s}$ \\
Chord lengths & $1.8 \mathrm{~m}, 27 \mathrm{~cm}$ \\
Temperature & $193 \mathrm{~K}$ \\
Reference pressure & $1 \mathrm{~atm}$ \\
Reference Length & $0.2 \mathrm{~m}$ \\
Reynolds number & $3,000,000 ; 341,400$ \\
\hline
\end{tabular}

COMSOL is used to generate mesh topology. Due to advantage of minimizing the skewness of a near wall mesh, $\mathrm{C}$-grid mesh is adopted to discrete flow field. A boundary layer mesh is applied with dense element distribution. Domain is optimized based on the number of cells and final mesh distribution which is used for this calculation is shown in Figure 2.

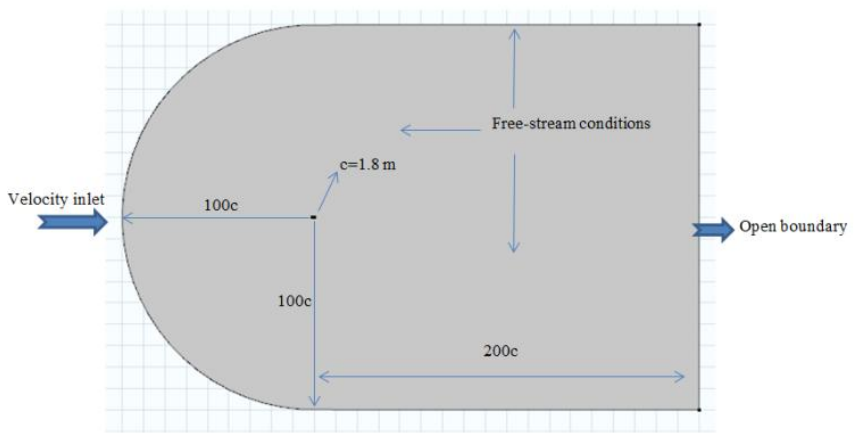

Fig. 1. Computational domain. 


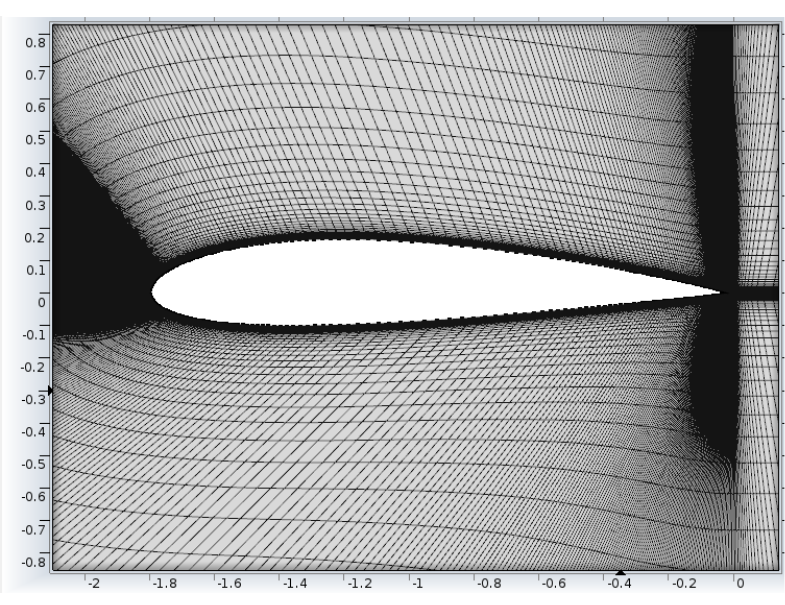

Fig. 2. Mesh distribution around airfoil.

\section{Results and Discussion}

The design of an efficient airplane requires careful balancing many conflicted requirement. This statement is especially true for airplane wing. To find optimum airfoil, NACA designed many different types of airfoil and tested 68 airfoils in wind tunnel in 1931 and published the result as a report [8]. The results were presented in the standard graphic form. Experimental test were conducted at the Reynolds numbers of 3,000,000. Another experiment was conducted in 2001 [2]. For the second experiment related with NACA 2415 airfoil was conducted at open circuit wind tunnels which pull the air from the environment into the tunnel and release the air back into the environment. Airfoil with $1.8 \mathrm{~m}$ and $26 \mathrm{~cm}$ chord length and $25 \mathrm{~m} / \mathrm{s}$ and $19.6 \mathrm{~m} / \mathrm{s}$ airflow speed were chosen for experiments with Reynolds numbers of 3,000,000 and 341,466. Experimental results are available as table data [2] and as a graph [8]. With the numerical simulation method, aerodynamic performance of NACA 2415 airfoil was analyzed to determine optimum angle of attack for maximum lift to drag ratio. Computational results were compared with the field experimental data of ref [2] and ref [8] for turbulent condition. Velocity magnitude and the streamlines for steady flow around the NACA 2415 airfoil at the angle of attack $10^{\circ}$ is shown in Figure 3. A location where the air flow speed increases is shown in red color in the graph. This means that low pressures is formed at the upper surface compared with bottom surface of airfoil and upwardly lift force is generated. The blue regions are the region where the lowest air flow dominates. These portions are also the location of turbulent flow. When the flow rate is increased, eddy current occurs in these areas.

Lift coefficients with different angle of attack and experimental results $[2,8]$ are shown in Figure 4 and Figure 5.With the increasing angle of attack lift coefficient increases linearly. There is discernible difference between the computational and experimental results. The agreement between the computational and experimental results is very good at the low angle but at high angles agreement is not good. This may be due to imperfection around airfoil section and experimental inaccuracies. A fully smooth blade profile in the experimental work is not always possible. But during theoretical calculations, all conditions are considered ideal in the framework of the parameters entered. Drag coefficient with different angle of attack were calculated and is shown in Figure 6. With the increasing angle, drag coefficient starts to degrease and reach minimum at zero angle of attack after then it starts to increase again and continue to increase with the angle.

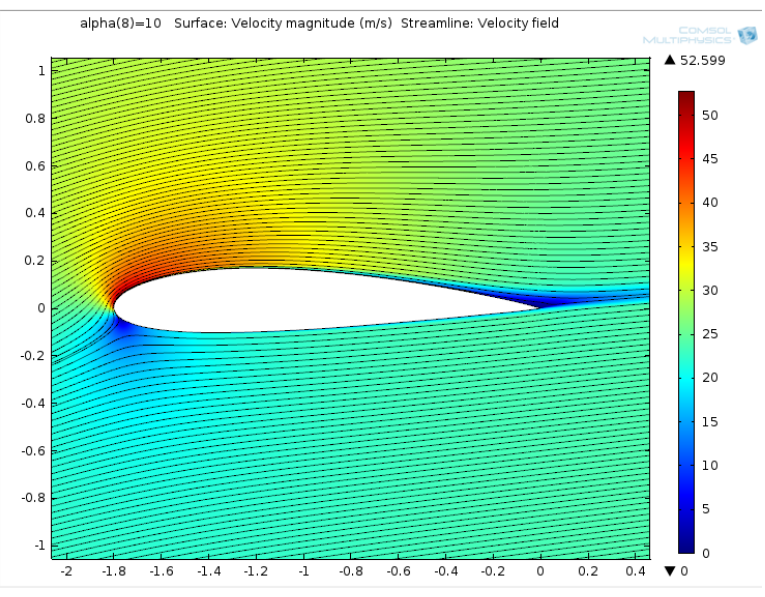

Fig. 3. Velocity magnitude and streamlines for the flow around a NACA 2415 airfoil.

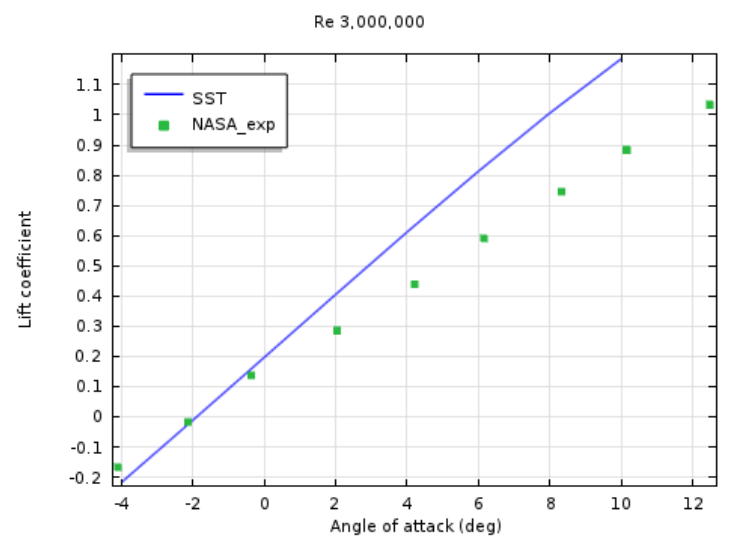

Fig. 4. Lift coefficient vs. angle of attack with NASA experimental data.

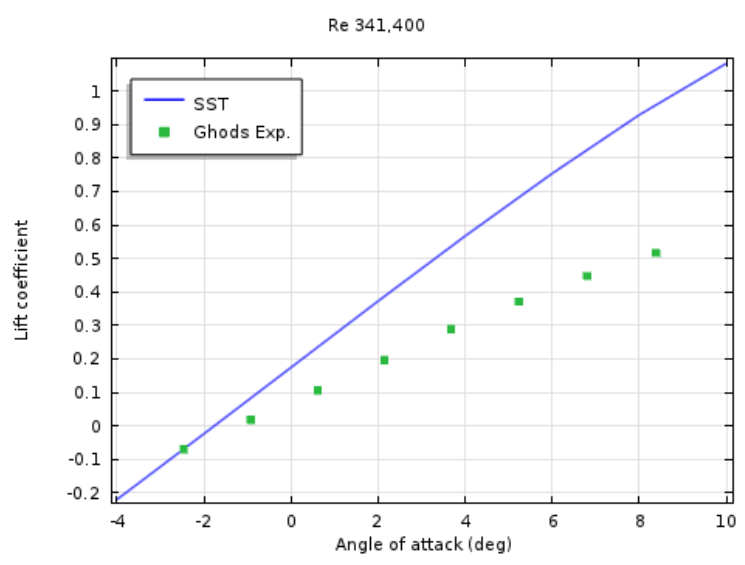

Fig. 5. Lift coefficient vs. angle of attack with Ghods experimental data. 


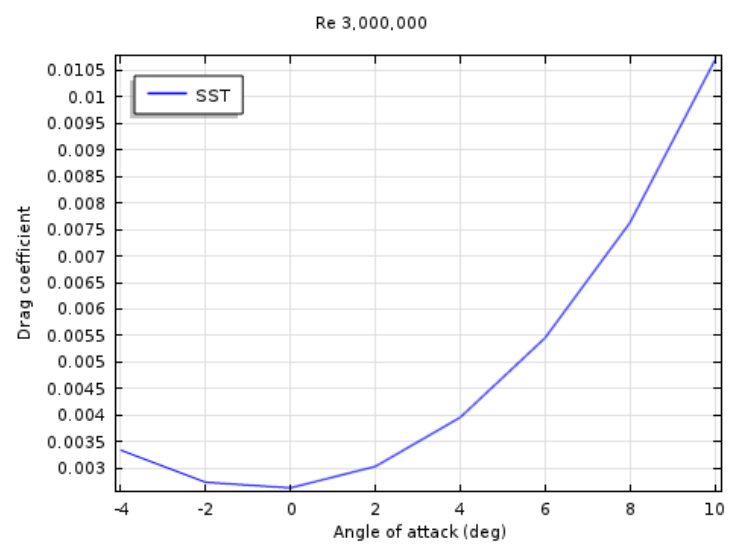

Fig. 6. Drag coefficient vs. angle of attack.

Lift to drag ratio was calculated by using SST turbulent model and is shown in Figure 7. Lift to drag ratio increases from $-4^{\circ}$ and becomes maximum at the angle of attack 4 degree then it start to degrease again. This figure indicates that optimum angle of attack for NACA 2415 airfoil is $4^{\circ}$ at low speed.

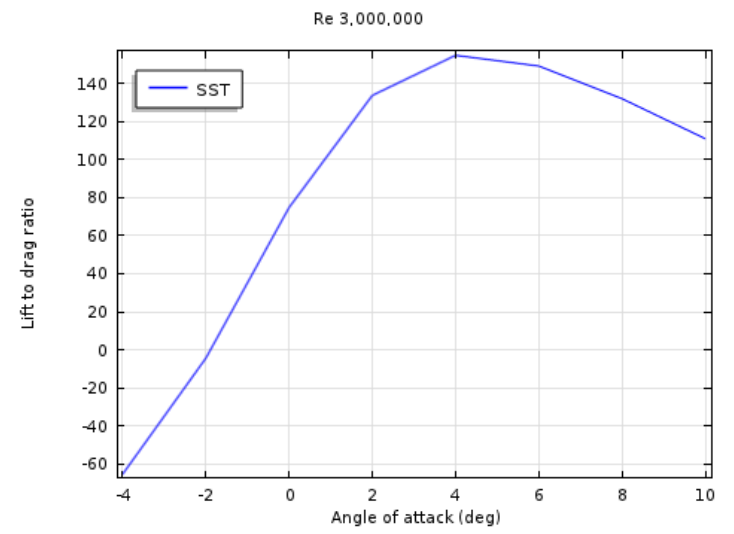

Fig. 7. Lift to drag ratio vs. angle of attack.

Pressure coefficient for NACA 2415 airfoil at the Reynolds numbers of 3,000,000 were calculated and are shown in Figure 8 for the angle of attack from $-4^{\circ}$ to $10^{\circ}$. With the increasing angle of attack from $-4^{\circ}$ to $10^{\circ}$, pressure difference between upper and lower surface increases. With the increasing angle, pressure decrement at the upper surface increases more quickly and pressure increment at the lower surface becomes slower.

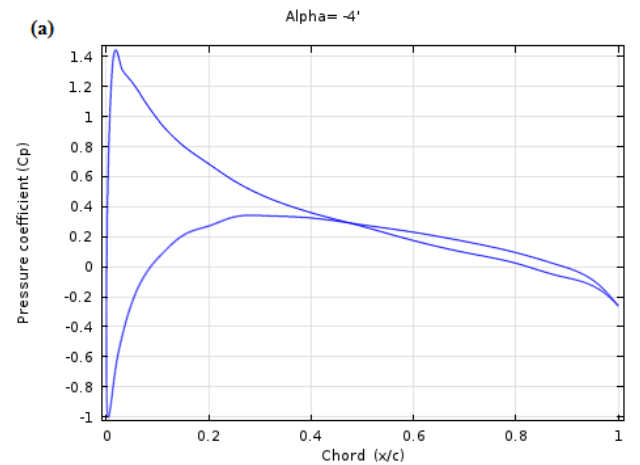

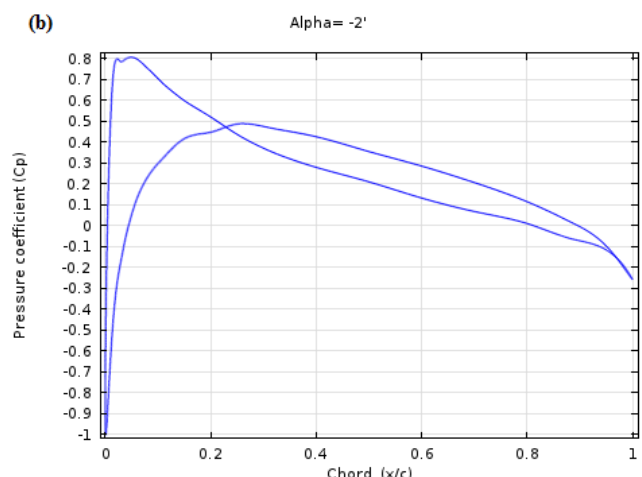
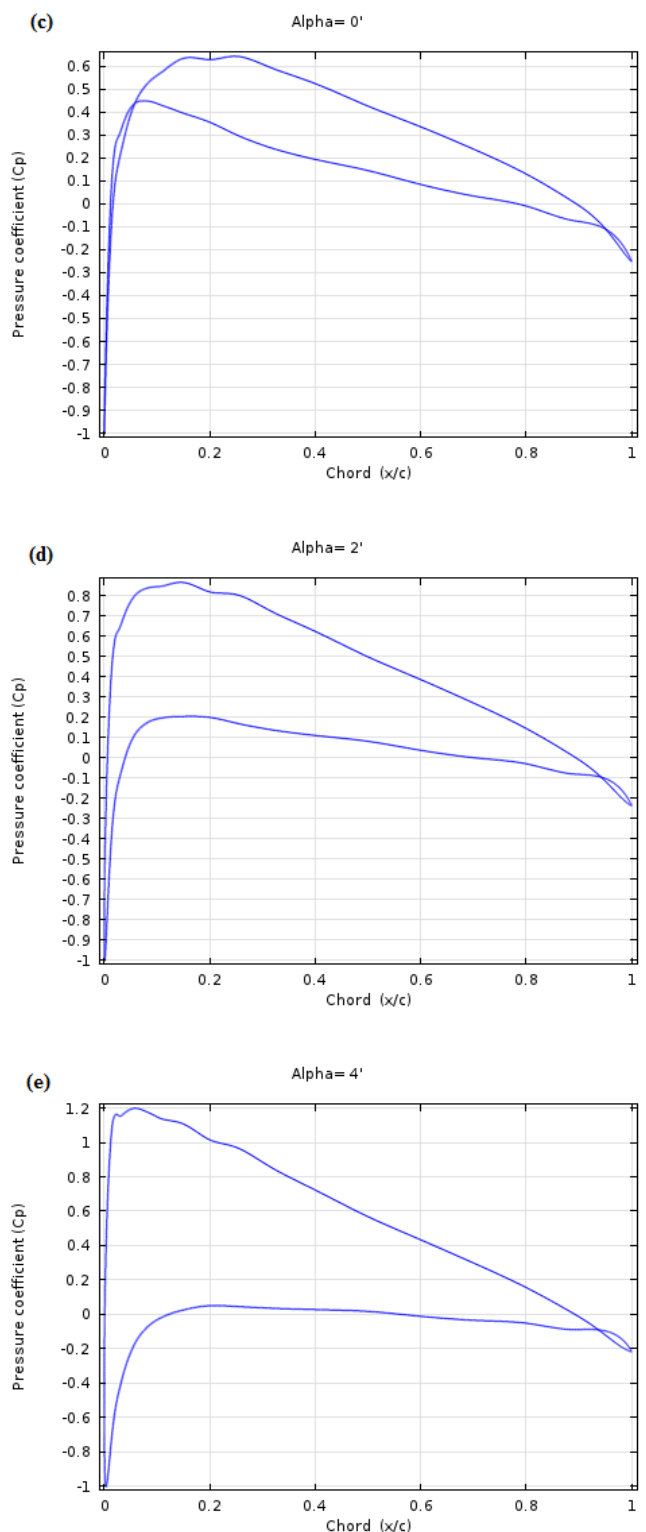

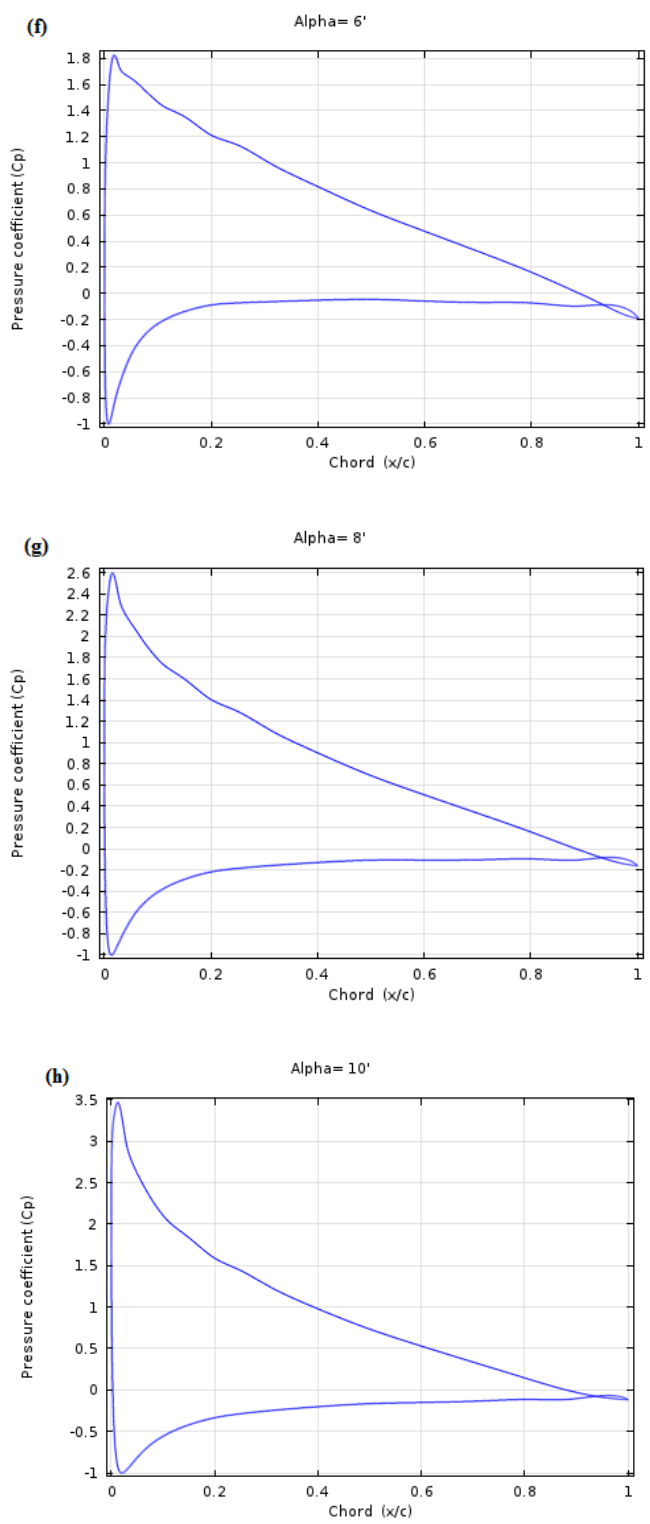

Fig. 8. Pressure coefficients of airfoil with different angle of attack.

\section{Conclusion}

Aircraft wings' horizontal and vertical stabilizers are built up with airfoil-shaped cross sections, as are helicopter rotor blades. Airfoils are also used in propellers, fans, compressors, wind turbines and almost all objects in returning. Sails are also made with the shape of airfoils, and the underwater surfaces of sailboats, are stand in crosssection and actuate on the same principles as airfoils. Swimming and flying creatures employ airfoils, such as bird wings, the bodies of fish etc. People first have made sharing information from birds to fly. In this study, effect of angle of attack on aerodynamic performance of airplane wing airfoil was conducted by CFD with SST turbulence model. Airfoil
NACA 2415 was simulated and results were compared with those two different wind tunnel experiment. The comparison shows good agreement at the low angle of attack but at high angle, agreement is not good with the experiment. Next, drag coefficient were calculated for different angle of attack and minimum drag coefficient were obtained at the angle of $0^{\circ}$. After then drag coefficient increases more quickly with the angle. Then, lift to drag ratios were calculated for different angle of attack from $-4^{\circ}$ to $10^{\circ}$ and maximum lift to drag ratio was found at the angle of attack $4^{\circ}$. Finally, pressure coefficient for the angle of attack from $-4^{\circ}$ to $10^{\circ}$ were calculated and analysed. With the increasing angle of attack, pressure difference between upper and lower surface increases with angle.

\section{Acknowledgment}

I thank Middle East Technical University, allowing me to this work in there with their facility.

\section{References}

[1] H.Sogukpinar, I. Bozkurt, "Calculation of Optimum Angle of Attack to Determine Maximum Lift to Drag Ratio of NACA 632-215 Airfoil”, Journal of Multidisciplinary Engineering Science and Technology, Vol. 2, pp. 1103-1108, 2015.

[2] M. Ghods, "Theory of Wings and Wind Tunnel Testing of A NACA 2415 Airfoil, Technical Communication for Engineers", The University of British Columbia, July 23, 2001.

[3] S. Rajakumar, D. Ravindran, "Iterative approach for optimizing coefficient of power, coefficient of lift and drag of wind turbine rotor", Renewable Energy, Vol. 38, pp. 83-93, 2012.

[4] A. Zanotti, R. Nilifard, G. Gibertini, A. Guardone, G. Quaranta, "Assessment of 2D/3D numerical modeling for deep dynamic stall experiments", Journal of Fluids and Structures, Vol. 51, pp. 97-115, 2014.

[5] M. J. Hoffmann, R. Reuss Ramsay, G.M. Gregorek, "Effects of Grit Roughness and Pitch Oscillations on the NACA 4415 Airfoil”, Airfoil Performance Report, NREL/TP-442-7815, 1996.

[6] C. Thumthae, T. Chitsomboon, "Optimal angle of attack for untwisted blade wind turbine", Renewable Energy, Vol. 34, pp. 1279-1284, 2009.

[7]COMSOL CFD module user guide http://www.comsol.com, 2015.

[8] N.J. Eastman, E.W. Kenneth, R.M Pinkerton, "The characteristics of 78 related airfoil sections from test in the variable-density wind tunnel", NACA report no: 460,1932 . 